\title{
Article
}

\section{Patient education interventions for the management of Inflammatory Bowel Disease}

Gordon, Morris, Sinopoulou, Vassiliki, Akobeng, Anthony K, Lakunina, Svetlana and Gjuladin-Hellon, Teuta

Available at http://clok.uclan.ac.uk/36586/

Gordon, Morris ORCID: 0000-0002-1216-5158, Sinopoulou, Vassiliki ORCID: 0000-0002-2831-9406, Akobeng, Anthony K, Lakunina, Svetlana ORCID: 00000002-3180-6336 and Gjuladin-Hellon, Teuta (2021) Patient education interventions for the management of Inflammatory Bowel Disease. Cochrane Database of Systematic Reviews .

It is advisable to refer to the publisher's version if you intend to cite from the work. http://dx.doi.org/10.1002/14651858.cd013854

For more information about UCLan's research in this area go to http://www.uclan.ac.uk/researchgroups/ and search for <name of research Group>.

For information about Research generally at UCLan please go to http://www.uclan.ac.uk/research/

All outputs in CLoK are protected by Intellectual Property Rights law, including Copyright law. Copyright, IPR and Moral Rights for the works on this site are retained by the individual authors and/or other copyright owners. Terms and conditions for use of this material are defined in the policies page.

\section{CLoK}

Central Lancashire online Knowledge www.clok.uclan.ac.uk

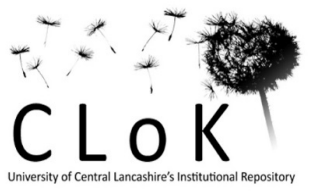


Cochrane Database of Systematic Reviews

\section{Patient education interventions for the management of Inflammatory Bowel Disease (Protocol)}

Gordon M, Sinopoulou V, Akobeng AK, Lakunina S, Gjuladin-Hellon T

Gordon M, Sinopoulou V, Akobeng AK, Lakunina S, Gjuladin-Hellon T.

Patient education interventions for the management of Inflammatory Bowel Disease (Protocol).

Cochrane Database of Systematic Reviews 2021, Issue 1. Art. No.: CD013854.

DOI: 10.1002/14651858.CD013854.

www.cochranelibrary.com

Patient education interventions for the management of Inflammatory Bowel Disease (Protocol) 
TABLE OF CONTENTS

HEADER 1

ABSTRACT

BACKGROUND

OBJECTIVES

METHODS

ACKNOWLEDGEMENTS

REFERENCES

APPENDICES

HISTORY

CONTRIBUTIONS OF AUTHORS

DECLARATIONS OF INTEREST 
[Intervention Protocol]

\section{Patient education interventions for the management of Inflammatory Bowel Disease}

Morris Gordon ${ }^{1}$, Vassiliki Sinopoulou ${ }^{1}$, Anthony K Akobeng ${ }^{2}$, Svetlana Lakunina ${ }^{1}$, Teuta Gjuladin-Hellon ${ }^{1}$

1School of Medicine, University of Central Lancashire, Preston, UK. 2Sidra Medicine, Doha, Qatar

Contact address: Morris Gordon, morris@betterprescribing.com, mgordon@uclan.ac.uk.

Editorial group: Cochrane Gut Group.

Publication status and date: New, published in Issue 1, 2021.

Citation: Gordon M, Sinopoulou V, Akobeng AK, Lakunina S, Gjuladin-Hellon T. Patient education interventions for the management of Inflammatory Bowel Disease (Protocol). Cochrane Database of Systematic Reviews 2021, Issue 1. Art. No.: CD013854. DOI: 10.1002/14651858.CD013854.

Copyright @ 2021 The Cochrane Collaboration. Published by John Wiley \& Sons, Ltd.

\section{A B S T R A C T}

\section{Objectives}

This is a protocol for a Cochrane Review (intervention). The objectives are as follows:

To identify the different types of educational interventions, how they are delivered, and to determine their effectiveness and safety in people with IBD. 


\section{B A C K G R O U N D}

\section{Description of the condition}

Inflammatory bowel disease (IBD) is an umbrella term for a range of conditions that cause inflammation to the human gastrointestinal tract, with the most prominent ones being ulcerative colitis and Crohn's disease. Symptoms can include pain, cramping, swelling, diarrhoea, weight loss and tiredness. The aetiology of IBD is still undetermined but it is thought to be caused via a complex interaction of genetic and environmental factors (De Souza 2017). More specifically, it is thought that IBD is due to an aberrant immune response to the gut commensal flora in a genetically susceptible individual (Pizarro 2019). IBD is a life-long condition for which currently there is no cure. Treatment options include medications, lifestyle and diet changes, and surgery with the aim of inducing and maintaining remission of the disease. It is estimated that more than 6.8 million people are living with IBD globally with incidences of the disease rising especially in regions that are newly adopting western lifestyles (Jairath 2020; Kaplan 2017). Apart from its physical manifestations, IBD can have a serious impact on patients' psychological and social well-being by limiting the patient's ability to take part in social activities and engagements. It also places a significant burden on healthcare systems, with an estimated EUR 4.6 billion to EUR 5.6 billion of annual healthcare costs attributed to IBD in Europe and USD 7.2 billion in the USA (Burisch 2013; Windsor 2019).

\section{Description of the intervention}

Patient educational interventions aim to deliver structured information to the recipient of the intervention and there is evidence to suggest patient education can have positive effects in other chronic diseases (Anderson 2017; Howcroft 2016). However, the content, delivery method, duration and specific purposes of any given intervention can vary considerably and there are no set standards for any of these parameters.

Local resources and healthcare systems, as well as individual patient factors, can have a major impact on patient education. Therefore, there is a need to understand whether such interventions can affect patient outcomes, and how and why they affect patient outcomes.

\section{How the intervention might work}

Education will enhance patient knowledge surrounding IBD. However, the question of how this may impact on their disease outcomes is complex. One point of focus has been about advising patients how to determine when their disease is deteriorating so they can contact their healthcare provider. Improving medication adherence, recognising adverse effects and when to report them, and improving compliance might be some of the ways patient education interventions might work.

IBD can affect patients' daily lives in several ways and can lead to a lower health-related quality of life. Together with physicianled management, self-management and knowledge about their disease can play an important role in giving patients control over their condition. IBD educational interventions can provide patients with important information and advice towards that end.

\section{Why it is important to do this review}

More clarity on the types of educational interventions targeting people with IBD that have been researched at a randomised controlled trial (RCT) level, what they entail and to what extent they are effective is vital for people with IBD to make better informed decisions for the self-management of their condition.

It is important to review systematically the evidence that has sought to address deficits identified in education (NRAD 2015), and to assess the attributes of training packages, so they can be applied effectively (Norcini 2011). The extent to which we can answer 'how' training can be designed, 'why' it is effective and 'for whom and when' will depend on descriptive data within primary studies, but it is important to highlight this information to help professionals understand and deliver health education in a reliable and reproducible manner (Gordon 2011; Gordon 2013).

\section{O B JECTIVES}

To identify the different types of educational interventions, how they are delivered, and to determine their effectiveness and safety in people with IBD.

\section{METHODS}

\section{Criteria for considering studies for this review}

\section{Types of studies}

All published, unpublished and ongoing RCTs that compare educational interventions targeted at people with IBD to any other type of intervention or no intervention.

\section{Types of participants}

People with IBD of all ages.

\section{Types of interventions}

Any type of formal or informal educational intervention, lasting for any time. Delivery methods can include face-to-face or remote educational sessions or workshops, guided study via the use of printed or online materials, the use of mobile applications or any other method that delivers information to patients.

We will list all intervention and comparator groups in the 'Characteristics of included studies' table.

\section{Types of outcome measures}

\section{Primary outcomes}

- Disease activity at study end, using a recognised disease activity scoring system as described by the study authors.

- Flare-ups or relapse measured clinically, endoscopically or histologically.

- Quality of life at study end using validated scales or tools.

\section{Secondary outcomes}

- Number of episodes accessing health care (outpatient, remote or inpatient.)

- Change in disease activity using a recognised score.

- Change in quality of life using a validated tool.

- Medication adherence. 
- Patient knowledge or skill (or both) as measured by a study. This will be specific assessment at study end (this outcome has a significant risk of heterogeneity that will probably prevent meta-analysis) and will be classified using Kirkpatrick's modified hierarchy, a model for analysing and evaluating the results of training and educational programmes (see 'Qualitative synthesis')

\section{Adverse effects}

- Total adverse effects (serious and minor).

- Adverse events leading to withdrawal.

\section{Qualitative synthesis}

We will record and synthesise the following to characterise educational interventions.

- Educational content (primary material, learning outcomes, theoretical underpinning).

- Teaching attributes of training programmes used (staff and resource requirements, length of course, methods including whether e-learning, asynchronous or synchronous, any followup service or session).

- Any knowledge assessment, including method used and reported pre- and post-test scores. Note if sufficiently homogeneous tests and education are deployed (such as standardised courses and testing materials), there may be scope for meta-analysis of test data. However, pilot searches performed suggest no such homogeneous methods exist and, therefore, it is not currently predicted that such analysis will possible. Therefore, a qualitative analysis is planned.

\section{Search methods for identification of studies}

\section{Electronic searches}

We will search the following sources to the date of search and will place no restrictions on the language of publication.

- Cochrane Central Register of Controlled Trials (CENTRAL; via Ovid, from inception) (Appendix 1).

- MEDLINE (via Ovid, from 1946) (Appendix 2).

- Embase (via Ovid, from 1974) (Appendix 3).

- PsycINFO (via Ovid, from 1987).

- CINAHL (Cumulative Index to Nursing and Allied Health Literature) (via EBSCO, from 1981).

- AMED (Allied and Complementary Medicine database) (via Ovid, from 1985).

- ClinicalTrials.gov (www.clinicaltrials.gov).

- World Health Organization International Clinical Trials Registry Platform (ICTRP) (www.who.int/trialsearch/).

\section{Searching other resources}

As complementary search methods, we will carefully check relevant systematic reviews for studies for potential inclusion in our review. In addition, we will scrutinise the references of included studies in our review. We will seek unpublished trials by contacting experts in the field and we will scan the Internet and abstracts submitted to major international congresses from the three years prior to the search to capture any studies presented but not yet published in full.
We will attempt to obtain translations of papers when necessary.

\section{Data collection and analysis}

We will carry out data collection and analysis according to the methods recommended in the Cochrane Handbook for Systematic Reviews of Interventions (Higgins 2020).

\section{Selection of studies}

Two review authors will independently screen the titles and abstracts identified from the literature search. We will discard studies that do not meet the inclusion criteria. We will then obtain the full report of studies that appear to meet our inclusion criteria or for which there is insufficient information to make a final decision. Two review authors will independently assess the reports to establish whether the studies meet the inclusion criteria. We will resolve disagreements by discussion, with a third review author consulted if resolution is not possible. We will enter studies rejected at this or subsequent stages in the 'Characteristics of excluded studies' tables and record the main reason for exclusion. We will record the selection process in sufficient detail to complete a PRISMA flow diagram.

Where studies have multiple publications, we will identify and exclude duplicates, and collate the reports of the same study so that each study, rather than each report, is the unit of interest for the review, and such studies have a single identifier with multiple references.

\section{Data extraction and management}

Two review authors will independently carry out data extraction using piloted data extraction forms. We will extract relevant data from full-text articles that meet the inclusion criteria including:

- trial setting: country and number of trial centres;

- methods: study design, total study duration and date;

- participant characteristics: age, sociodemographics, ethnicity, diagnostic criteria and total number;

- eligibility criteria: inclusion and exclusion criteria;

- intervention and comparator - this will include description of the learning outcomes planned for the intervention by the teacher or designer, methods of education used, target audience and any resources required);

- patient outcomes: patient outcome definition, unit of measurement and time of collection;

- outcomes from education: educational outcomes, if described, reported and classified as either satisfaction/reaction, attitudes or knowledge and skills;

- results: number of participants allocated to each group, missing participants, sample size;

- funding source.

\section{Assessment of risk of bias in included studies}

During data extraction, two review authors will independently assess all studies meeting the inclusion criteria for their risk of bias using criteria outlined in the Cochrane Handbook for Systematic Reviews of Interventions (Higgins 2020). The domains that we will assessed are as follows.

- Sequence generation (selection bias). 
- Allocation concealment (selection bias).

- Blinding of participants and personnel (performance bias).

- Blinding of outcome assessment (detection bias).

- Incomplete outcome data (attrition bias).

- Selective reporting (reporting bias).

- Other bias.

We will judge the studies to be at low, high or unclear risk of bias for each domain assessed, based on guidance in the Cochrane Handbook for Systematic Reviews of Interventions (Higgins 2020).

After data extraction, the two review authors will compare the extracted data to discuss and resolve discrepancies before the data are transferred into the 'Characteristics of included studies' table. For cluster RCTs, we intend to judge risk of bias as prescribed in section 23.1.2 "Assessing risk of bias in cluster-randomized trials" of the Cochrane Handbook for Systematic Reviews of Interventions (Higgins 2020).

\section{Measures of treatment effect}

For dichotomous outcomes, we will express treatment effect as risk ratios (RR) with corresponding 95\% confidence intervals (Cls). For continuous outcomes, we will express the treatment effect as mean difference (MD) with $95 \% \mathrm{Cl}$ if studies use the same scales and methods. However, if studies assess the same continuous outcome using different methods, we will estimate the treatment effect using the standardised mean difference (SMD) with 95\% Cls. We will present SMDs as standard deviation units and interpret them as follows: 0.2 represents a small effect, 0.5 a moderate effect and 0.8 a large effect.

\section{Unit of analysis issues}

The participant will be the unit of analysis. For studies comparing more than two intervention groups, we will make multiple pairwise comparisons between all possible pairs of intervention groups. To avoid double counting, we intend to divide out shared intervention groups evenly among the comparisons. For dichotomous outcomes, we plan to divide up both the number of events and the total number of participants. For continuous outcomes, we will divide up the total number of participants and leave the means and standard deviations unchanged. We will include cross-over studies if data are separately reported before and after cross-over and we will only use data from the first phase for our analysis. We do not anticipate finding any cluster RCTs; however, we will use study data only if the authors have used appropriate statistical methods in taking clustering effect into account. We will also exclude cluster RCTs from a sensitivity analysis to assess their impact on the results.

\section{Dealing with missing data}

We aim to contact authors where there are missing data or studies have not reported data in sufficient detail. We will attempt to estimate missing standard deviations using relevant statistical tools and calculators if studies report standard errors. Studies that fail to report measures of variance will be judged as being at high risk of selective reporting bias.

\section{Assessment of heterogeneity}

We will scrutinise studies to ensure that they are clinically homogeneous in terms of participants, intervention, comparator and outcome. To test for statistical heterogeneity, we will use a Chi2 test. A P value of less than 0.1 will give an indication of the presence of heterogeneity. Inconsistency will be quantified and represented by the $1^{2}$ statistic. The thresholds will be interpreted as follows (Higgins 2020):

- $0 \%$ to $40 \%$ : might not be important;

- $30 \%$ to $60 \%$ : may represent moderate heterogeneity;

- $50 \%$ to $90 \%$; may represent substantial heterogeneity;

- $75 \%$ to $100 \%$ : considerable heterogeneity.

\section{Assessment of reporting biases}

Most reporting biases will be minimised by using an inclusive search strategy. We will investigate publication bias using a funnel plot if there are 10 or more studies. The magnitude of publication bias will be determined by visual inspection of the asymmetry of the funnel plot. In addition, we will test funnel plot asymmetry by performing a linear regression of intervention effect estimate against its standard error, weighted by the inverse of the variance of the intervention effect estimate (Egger 1997).

\section{Data synthesis}

To summarise the study characteristics, we intend to conduct a narrative synthesis of all the included studies. We will then carry out a meta-analysis if there are two more studies that have assessed similar populations, interventions and outcomes. We will analyse studies from paediatric populations, adult populations and different subintervention types separately. We will synthesise data using the random-effects model in Review Manager 5 (Review Manager 2014). We will combine effect estimates of studies which report data in a similar way, in the meta-analysis. We will pool RRs for dichotomous outcomes and MDs or SMDs for continuous outcomes with $95 \% \mathrm{Cls}$. Where we are unable to carry out a metaanalysis (e.g. due to lack of uniformity in data reporting), we will present a narrative summary of the included studies.

\section{Subgroup analysis and investigation of heterogeneity}

If there is heterogeneity, we will investigate possible causes and address them using methods described in the Cochrane Handbook for Systematic Reviews of Interventions (Higgins 2020). We will undertake subgroup analyses of potential effect modifiers if there are 10 studies or more. If enough data are available, we will perform subgroup analyses by age, gender and disease type.

It is recognised that the nature of the studies likely to be included in this review may be capricious and heterogeneous in a number of key clinical and methodological ways that cannot be fully predicted. If such factors are identified and become relevant to ensure integrity of the analysis, modifications to this list may be needed. These will be fully reported by the authors.

\section{Sensitivity analysis}

Where possible, we plan to undertake a sensitivity analysis on the primary outcome of 'disease activity at study end', to assess whether the findings of the review are robust to the decisions made during the review process. In particular, we intend to exclude studies at high or unclear risk of bias due to allocation bias and performance bias from analyses that have a mix of studies with different risk of bias judgements. Where data analyses include studies with reported and estimated standard deviations, we plan 
to exclude those with estimated standard deviations to assess whether this affects the findings of the review. We will investigate whether the choice of model (fixed versus random) impacts the results to explore heterogeneity.

\section{Summary of findings and assessment of the certainty of the evidence}

We will present the main results in a 'Summary of findings' table. Each comparison and primary outcome will be exported to GRADEprofiler software (developed by the GRADE Working Group) for quality assessment (GRADE 2015). We will include all three primary outcomes. We will apply GRADE to any additional comparisons and present these in additional tables. Based on risk of bias, inconsistency, imprecision, indirectness and publication bias, we will rate the certainty of the evidence for each outcome as high, moderate, low or very low. These ratings have been defined as follows.

- High: further research is very unlikely to change our confidence in the estimate of effect.

- Moderate: further research is likely to have an important impact on our confidence in the estimate of effect and may change the estimate.
- Low: further research is very likely to have an important impact on our confidence in the estimate of effect and is likely to change the estimate.

- Very low: any estimate of effect is very uncertain.

We will justify all decisions to downgrade the quality of studies using footnotes and we will make comments to aid reader's understanding of the review where necessary.

\section{A C K N O WLEDGEMENTS}

Authors acknowledge the following reviewers: Dr Daniel Gaya (Consultant Gastroenterologist, Glasgow Royal Infirmary, Glasgow, UK) and Dr Stephen J Bickston (Medical Director, Center for Inflammatory Bowel Diseases, Virginia Commonwealth University Health System, USA), and consumer reviewer Ms Kim Daley.

Authors acknowledge the copy editor, Anne Lawson.

Yuhong Yuan (Information Specialist at the Cochrane Gut Group) designed the search strategies. 


\section{RE F E R E N C E S}

\section{Additional references}

\section{Anderson 2017}

Anderson L, Brown JP, Clark AM, Dalal H, Rossau HK, Bridges C, et al. Patient education in the management of coronary heart disease. Cochrane Database of Systematic Reviews 2017, Issue 6. Art. No: CD008895. [DOI: 10.1002/14651858.CD008895.pub3]

\section{Burisch 2013}

Burisch J, Jess T, Martinato M, Lakatos PL. The burden of inflammatory bowel disease in Europe. Journal of Crohn's and Colitis 2013;7(4):322-37.

\section{De Souza 2017}

De Souza HS, Fiocchi C, Iliopoulos D. The IBD interactome: an integrated view of aetiology, pathogenesis and therapy. Nature Reviews. Gastroenterology \& Hepatology 2017;14(12):739-49.

\section{Egger 1997}

Egger M, Smith GD, Minder C. Bias in meta-analysis detected by a simple, graphical test. BMJ 1997;315(7109):629-34. [DOI 10.1136/bmj.315.7109.629]

\section{Gordon 2011}

Gordon M, Findley R. Educational interventions to improve handover in health care: a systematic review. Medical Education 2011;45(11):1081-9.

\section{Gordon 2013}

Gordon M. Non-technical skills training to enhance patient safety. Clinical Teacher 2013;10:170-5.

\section{GRADE 2015 [Computer program]}

McMaster University (developed by Evidence Prime) GRADEpro GDT. Hamilton (ON): McMaster University (developed by Evidence Prime), 2015. Available at gradepro.org.

\section{Higgins 2020}

Higgins JPT, Thomas J, Chandler J, Cumpston M, Li T, Page MJ, Welch VA (editors). Cochrane Handbook for Systematic Reviews of Interventions version 6.1 (updated September 2020). Cochrane, 2020. Available from www.training.cochrane.org/ handbook.

\section{AP PE N DICES}

\section{Appendix 1. CENTRAL search strategy (Ovid)}

1. exp Inflammatory bowel diseases/

2. (inflammatory bowel disease* or IBD).tw,kw.

3. $\mathrm{crohn}^{*} . \mathrm{tw}, \mathrm{kw}$.

4. (colitis or regional enteritis or proctocolitis or colorectitis).tw,kw.

5. (UC or CD).tw,kw.

6. or $/ 1-5$

7. exp patient education handout/

8. exp Education/

9. exp patient medication knowledge/

\section{Howcroft 2016}

Howcroft M, Walters EH, Wood-Baker R, Walters JA. Action plans with brief patient education for exacerbations in chronic obstructive pulmonary disease. Cochrane Database of Systematic Reviews 2016, Issue 12. Art. No: CD005074. [DOI: 10.1002/14651858.CD005074.pub4]

\section{Jairath 2020}

Jairath V, Feagan BG. Global burden of inflammatory bowel disease. Lancet Gastroenterology \& Hepatology 2020;5(1):2-3.

\section{Kaplan 2017}

Kaplan GG, Ng SC. Understanding and preventing the global increase of inflammatory bowel disease. Gastroenterology 2017;152(2):313-21.

\section{Norcini 2011}

Norcini J, Anderson B, Bollela V, Burch V, Costa MJ, Duvivier R, et al. Criteria for good assessment: consensus statement and recommendations from the Ottawa 2010 conference. Medical Teacher 2011;33(3):206-14.

\section{NRAD 2015}

National Review of Asthma Deaths. Why asthma still kills, 2015. www.rcplondon.ac.uk/projects/outputs/why-asthma-still-kills (accessed 21 July 2020).

\section{Pizarro 2019}

Pizarro TT, Stappenbeck TS, Rieder F, Rosen MJ, Colombel JF, Donowitz $\mathrm{M}$, et al. Challenges in IBD research: preclinical human IBD mechanisms. Inflammatory Bowel Diseases 2019;25(Suppl 2):S5-12.

\section{Review Manager 2014 [Computer program]}

Nordic Cochrane Centre, The Cochrane Collaboration Review Manager (RevMan). Version 5.3. Copenhagen: Nordic Cochrane Centre, The Cochrane Collaboration, 2014.

\section{Windsor 2019}

Windsor JW, Kaplan GG. Evolving epidemiology of IBD. Current Gastroenterology Reports 2019;21(8):40. 
10. ((patient ${ }^{\star}$ or consumer $\left.{ }^{\star}\right)$ adj3 (educat ${ }^{\star}$ or literacy or training or workshop* or handout or session* or printed or print online or internet or booklet ${ }^{\star}$ or poster ${ }^{\star}$ or paper-based or pamphlet ${ }^{\star}$ or brochure* or leaflet* or circular $^{\star}$ or flyer ${ }^{\star}$ or program ${ }^{\star}$ or teaching or knowledge or information)).tw,kw.

11.((information or education*) adj3 (workshop* or handout or session* or printed or print or online or internet or booklet* or poster* or written material ${ }^{\star}$ or paper-based or pamphlet ${ }^{\star}$ or brochure ${ }^{\star}$ or leaflet ${ }^{\star}$ or circular ${ }^{\star}$ or flyer $^{\star}$ or program ${ }^{\star}$ or knowledge)).tw,kw.

12.((print or printed or education*) adj2 intervention*).tw,kw.

13. (health education or health information or health literacy).tw, kw.

14.or/7-13

15.6 and 14

\section{Appendix 2. MEDLINE search strategy (Ovid)}

1. exp Inflammatory bowel diseases/

2. (inflammatory bowel disease* or IBD).tw,kw.

3. crohn*.tw,kw.

4. (colitis or regional enteritis or proctocolitis or colorectitis).tw,kw.

5. (UC or CD).tw,kw.

6. or/1-5

7. exp patient education handout/

8. exp Education/

9. exp patient medication knowledge/

10. ((patient* or consumer $\left.^{\star}\right)$ adj3 (educat ${ }^{\star}$ or literacy or training or workshop* or handout or session* or printed or print online or internet or booklet ${ }^{\star}$ or poster ${ }^{\star}$ or written material ${ }^{\star}$ or paper-based or pamphlet ${ }^{\star}$ or brochure $^{\star}$ or leaflet ${ }^{\star}$ or circular ${ }^{\star}$ or flyer $^{\star}$ or program $^{\star}$ or teaching or knowledge or information)).tw,kw.

11.((information or education $\left.{ }^{\star}\right)$ adj3 (workshop* or handout or session ${ }^{\star}$ or printed or print or online or internet or booklet ${ }^{\star}$ or poster ${ }^{\star}$ or written material ${ }^{\star}$ or paper-based or pamphlet ${ }^{\star}$ or brochure ${ }^{\star}$ or leaflet ${ }^{\star}$ or circular $^{\star}$ or flyer ${ }^{\star}$ or program ${ }^{\star}$ or knowledge)).tw,kw.

12.((print or printed or education $\left.{ }^{\star}\right)$ adj2 intervention $\left.{ }^{\star}\right)$.tw,kw.

13. (health education or health information or health literacy).tw,kw.

14.or/7-13

15.6 and 14

16. randomized controlled trial.pt.

17.controlled clinical trial.pt.

18. random*.ab.

19.placebo.ab.

20.trial.ab.

21.groups.ab.

22.or/16-21

23.exp animals/ not humans.sh.

24.22 not 23

25.15 and 24

Note: Lines 16-24. RCT filter: Cochrane Highly Sensitive Search Strategy for identifying randomised trials in MEDLINE: sensitivitymaximising version (2008 revision); Ovid format" We made the following minor revisions: we used "random " instead of "randomized.ab" or "randomly.ab." to capture word variations such as "randomised, randomization, random"; we removed "drug therapy.fs." from the above filter as this review is not related to drug therapy.

\section{Appendix 3. Embase search strategy (Ovid)}

1. exp inflammatory bowel disease/

2. (inflammatory bowel disease* or IBD).tw,kw.

3. crohn*.tw,kw.

4. (colitis or regional enteritis or proctocolitis or colorectitis).tw,kw.

5. (UC or CD).tw,kw.

6. $\mathrm{or} / 1-5$

7. exp education/ 
8. ((patient ${ }^{\star}$ or consumer ${ }^{\star}$ ) adj3 (educat ${ }^{\star}$ or literacy or training or workshop* or handout or session* or printed or print online or internet or booklet ${ }^{\star}$ or poster ${ }^{\star}$ or written material ${ }^{\star}$ or paper-based or pamphlet ${ }^{\star}$ or brochure $^{\star}$ or leaflet ${ }^{\star}$ or circular ${ }^{\star}$ or flyer $^{\star}$ or program $^{\star}$ or $^{*}$ teaching or knowledge or information)).tw,kw.

9. ((information or education*) adj3 (workshop* or handout or session* or printed or print or online or internet or booklet* or poster* or written material ${ }^{\star}$ or paper-based or pamphlet ${ }^{\star}$ or brochure ${ }^{\star}$ or leaflet ${ }^{\star}$ or circular $^{\star}$ or flyer $^{\star}$ or program ${ }^{\star}$ or knowledge)).tw,kw.

10. ((print or printed or education*) adj2 intervention*).tw,kw.

11. (health education or health information or health literacy).tw,kw.

12.or/7-11

13.6 and 12

14.random:.tw.

15.placebo:.mp.

16. double-blind:.tw.

17.or/14-16

18.exp animal/ not human/

19.17 not 18

20.13 and 19

Lines \#14-16, RCT filter. Hedge Best balance of sensitivity and specificity filter for identifying randomised trials in Embase. hiru.mcmaster.ca/hiru/HIRU_Hedges_EMBASE_Strategies.aspx

\section{HISTORY}

Protocol first published: Issue 1, 2021

\section{CONTRIBUTIONS OF AUTHORS}

Morris Gordon: conceived the review question; secured funding and developed the review; performed screening of titles \& abstracts and full text articles, data extraction and contacted authors, analysed and interpreted data; checked quality assessment; checked the quality of statistical analysis; contributed to writing and editing the review; made an intellectual contribution to, advised on, approved the final version prior to submission; and is a guarantor of the protocol.

Vassiliki Sinopoulou: extracted data and contacted authors; checked the quality of data extraction; analysed and interpreted data; undertook and checked quality assessment; performed statistical analysis; checked the quality of the statistical analysis; produced the first draft of the review; contributed to writing and editing the review; made an intellectual contribution to the review; approved the final review prior to submission.

Anthony Akobeng:

\section{DECLARATIONS OF INTEREST}

MG: since August 2016, I have received travel fees to attend international scientific and training meetings from Pharma companies. These grants included no honoraria, inducement, advisory role or any other relationship and were restricted to the travel and meeting related costs of attending such meetings. These included: DDW (Digestive Disease Week) May 2017, World Congress of Gastroenterology October 2017, DDW May 2018, Advances in IBD December 2018 and DDW May 2019. The companies included: Biogaia (2017 to 2019), Ferring (2018), Allergan (2017), Synergy (bankrupt in 2018) and Tillots (2017 to 2019). None of these companies have had any involvement in any works completed by me and I have never had any payments for any other activities for them, as confirmed below. From these dates onwards, I have made a personal undertaking to take no further funds from any pharmaceutical or formula company in any form for travel or other related activities. This is to lift the limitations such funding has on my ability to act as a first and corresponding author on reviews, in line with the Cochrane policies on such matters and is reported in line with these policies. These current declarations will expire over the next three years (by 2023) and this statement will be updated regularly to reflect this.

VS: none.

AKA: none.

SL: none.

TGH: none. 